\title{
Automated diagnostics for resonance signature recognition on IMAGE/RPI plasmagrams
}

\author{
Ivan A. Galkin, Bodo W. Reinisch, and Xueqin Huang \\ Center for Atmospheric Research, University of Massachusetts, Lowell, Massachusetts, USA
}

Robert F. Benson

Laboratory for Extraterrestrial Physics, NASA Goddard Space Flight Center, Greenbelt, Maryland, USA

Shing F. Fung

Space Physics Data Facility, NASA Goddard Space Flight Center, Greenbelt, Maryland, USA

Received 6 June 2003; revised 25 November 2003; accepted 17 December 2003; published 24 February 2004.

[1] The Radio Plasma Imager (RPI) aboard the IMAGE spacecraft probes plasma at both far and near ranges by means of radio sounding. The RPI plasmagrams, similar in their concept to the ground-based and topside ionograms, contain not only a variety of signatures pertaining to the remote plasma structures and boundaries, but also a suite of the local plasma resonances stimulated by the RPI radio transmissions. Detection and interpretation of the resonance signatures is a valuable diagnostic tool providing the actual electron density and magnetic field strength at the spacecraft location, which are needed for the accurate processing of the remote sensing information on the plasmagrams. The high volume of the RPI sounding data demanded the development of automated techniques for routine interpretation of the plasmagrams. This paper discusses a new method for the detection and interpretation of the resonance signatures in the RPI plasmagrams that employs pattern recognition techniques to localize the signatures and identifies them in relation to model-based resonances. INDEX TERMS: 2494 Ionosphere: Instruments and techniques; 2794 Magnetospheric Physics: Instruments and techniques; 6994 Radio Science: Instruments and techniques; 7819 Space Plasma Physics: Experimental and mathematical techniques; KEYWORDS: intelligent systems, radio sounding, Radio Plasma Imager

Citation: Galkin, I. A., B. W. Reinisch, X. Huang, R. F. Benson, and S. F. Fung (2004), Automated diagnostics for resonance signature recognition on IMAGE/RPI plasmagrams, Radio Sci., 39, RS1015, doi:10.1029/2003RS002921.

\section{Introduction}

[2] A radio transmitter immersed in plasma is capable of stimulating short-range plasma-wave echoes and plasma emissions if its sounding frequency matches one of the characteristic frequencies of the plasma, often called resonance frequencies, or simply resonances. Typical resonance signatures have been observed by a number of space missions carrying a topside ionosonde or a relaxation sounder (see, e.g., Muldrew [1972] for a representative review). Detection of stimulated resonances and wave cutoffs in the radio sounding data provides a measurement of local plasma

Copyright 2004 by the American Geophysical Union. 0048-6604/04/2003RS002921\$11.00 density and magnetic field that has demonstrated accuracy and diagnostic potential superior to what conventional magnetometers and density probes achieve.

[3] The Radio Plasma Imager (RPI) [Reinisch et al., 2000] on the IMAGE spacecraft [Burch, 2000] is the first radio sounder ever flown on a highly elliptical orbit into the magnetosphere. It employs the stepped-frequency radio sounding concept that has been successfully practiced for several decades in a variety of applications [e.g., Reinisch, 1996; Pulinets and Benson, 1999]. Figure 1 is the BinBrowser virtual range versus frequency presentation of the received RPI signal amplitudes, called a plasmagram [Reinisch et al., 2000]. The virtual range is simply one half the echo delay time multiplied by the free-space speed of light. The pixel colors in the plasmagram image in Figure 1 represent the logarithmic amplitudes of the signals received on the $\mathrm{Z}$ antenna, calculated 


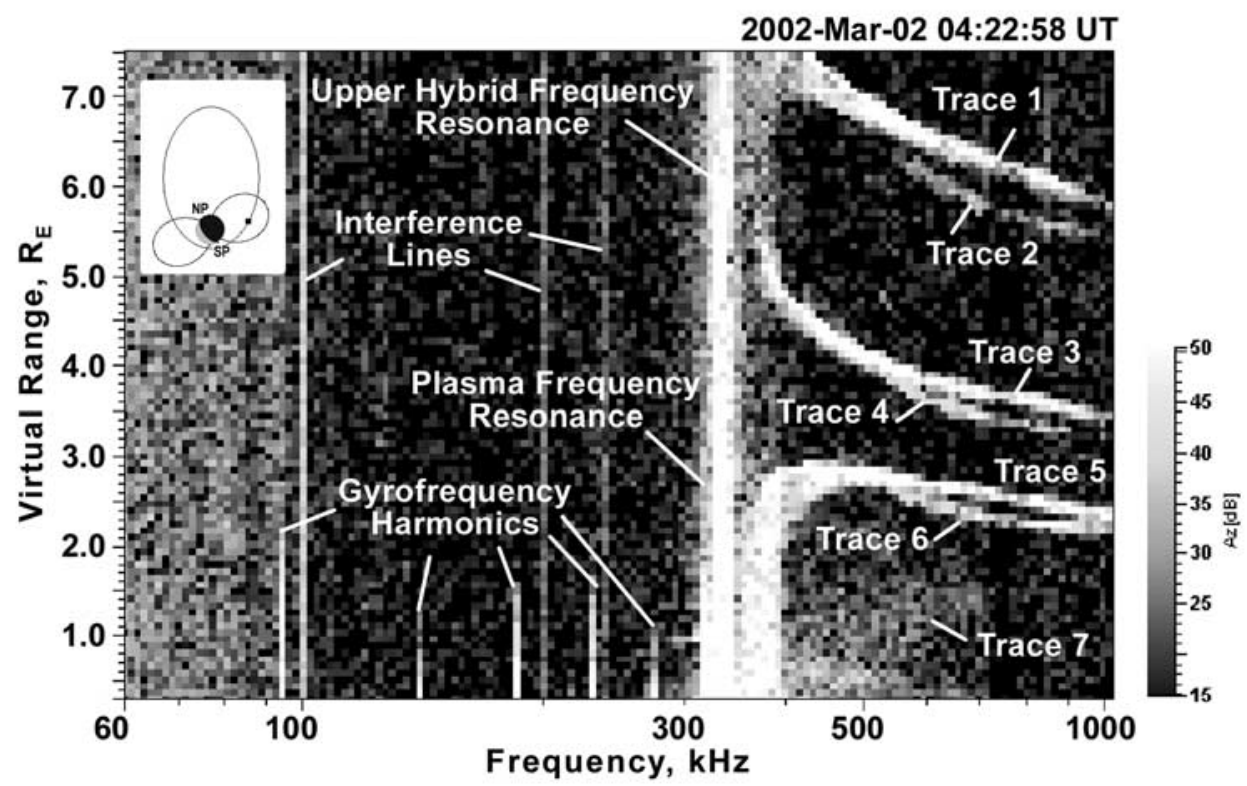

Figure 1. RPI Plasmagram on 2 March 2002, 04:23 UT with remote echo traces and resonance signatures as indicated. See color version of this figure at back of this issue.

from the recorded 12-bit in-phase and quadrature voltage samples.

[4] The sample plasmagram in Figure 1 shows two types of signatures: (1) traces, formed by the RPI echoes reflecting from remote locations in the plasma, and (2) resonances, appearing as vertical line segments extending upward from zero virtual range, each corresponding to a particular resonant response of the local plasma to the transmitter pulse. We have identified five major types of resonances in the RPI plasmagrams, all pertaining to oscillations of the plasma electrons. They are observed at the following frequencies: (a) electron cyclotron frequency $f_{\text {ce }}$, and its harmonics, $n \cdot f_{\text {ce }}$, (b) electron plasma frequency $f_{\mathrm{pe}}$, (c) upper-hybrid frequency $f_{\mathrm{uh}}$, (d) Qn resonances $f_{\text {Qn }}$ (also known as Bernstein-mode resonances), and (e) Dn resonances $f_{\text {Dn }}$.

[5] A number of dependencies exist between the plasma resonance frequencies [Stix, 1962]. The upperhybrid frequency, $f_{\text {uh }}$, is given by the plasma and gyro frequencies:

$$
f_{u h}=\sqrt{f_{p e}^{2}+f_{c e}^{2}}
$$

The Qn resonances can be related to the plasma and gyro frequencies by an approximate expression [Warren and Hagg, 1968]:

$$
f_{Q n} \approx f_{c e}\left[n+\frac{0.46}{n^{2}} \frac{f_{p e}^{2}}{f_{c e}^{2}}\right],
$$

which is a good approximation when $\mathrm{f}_{\mathrm{Qn}} / \mathrm{f}_{\mathrm{ce}}$ is near integer values. Benson et al. [2001] provided curves for $\mathrm{f}_{\mathrm{Qn}} / \mathrm{f}_{\mathrm{ce}}$ based on electrostatic dispersion equation solutions for zero-group velocity plasma waves perpendicular to the magnetic field. Finally, the sequence of Dn resonances is described by the following expressions [Osherovich and Benson, 1991]:

$$
\begin{array}{r}
f_{D n}=0.95 \sqrt{f_{p} f_{c e}} \sqrt{n} \\
f_{D n}^{+}=\sqrt{f_{D n}^{2}+f_{c e}^{2}} \\
f_{D n}^{-}=\sqrt{f_{D n}^{2}-f_{c e}^{2}}
\end{array}
$$

Equations (1)-(3) can be used to build a composite model of the resonance signatures driven only by the gyrofrequency $f_{\text {ce }}$ and the plasma frequency $f_{\mathrm{pe}}$.

\section{Review of Previous Automated Resonance Recognition Work}

[6] Interpretation of the resonance signatures usually requires matching of the observed signatures to model values. This involves a certain amount of simple calculations that are time consuming to carry out manually. Automatic resonance matching algorithms have been developed since the early 1980s, all fitting the resonance model to the data by testing a number of candidate values 
of $f_{\text {ce }}$ and $f_{\text {pe }}$ and selecting the best fit values. Huang and Reinisch [1982] and Huang et al. [2002] developed an automated resonance detection algorithm that is now a part of the TOPIST system for autoscaling topside ionograms from the Alouette and ISIS sounders (http://nssdc.gsfc. nasa.gov/space/isis/isis-status.html). The algorithm seeks the "best" combination of the X-mode cutoff frequency and the gyrofrequency that maximizes the amplitude sum over range bins extending to $500 \mathrm{~km}$ on 5 frequencies: (1) O-mode cutoff or local plasma frequency $f_{\text {pe }}$, (2) X-mode cutoff frequency $f_{\mathrm{x}},(3)$ upper hybrid frequency $f_{\mathrm{uh}}$, and (4) and (5) two neighboring gyrofrequency harmonics $\mathrm{n} f_{\text {ce }}$ and $(\mathrm{n}+1) f_{\text {ce }}$, selected to be close to the other resonances within the ionogram image. Igi et al. [1982] developed a similar algorithm for the topside sounder on the ISS-B spacecraft using two separate fits, one for the gyrofrequency $f_{\text {ce }}$, and another for the triplet $f_{\mathrm{pe}}, f_{\mathrm{uh}}, f_{\mathrm{x}}$.

[7] Trotignon et al. [1986] reported successful algorithmic solutions to the task of extracting resonance signatures from the ISEE-1 relaxation sounder data. The ISEE-1 satellite had a highly elliptic orbit with an apogee of $\sim 23 \mathrm{R}_{\mathrm{E}}$ (Earth's radius $=6,375 \mathrm{~km}$ ) and a perigee of $280 \mathrm{~km}$ and therefore observed a wide range of plasma densities in the solar wind, magnetosheath, magnetosphere, and magnetospheric tail. The resonance signatures in the magnetosphere were found to exhibit the greatest variety and present the greatest challenge to automated recognition. As a definite advantage for the automated data analysis, the ISEE-1 had an onboard magnetometer to measure the value of the gyrofrequency with an accuracy of $\sim 1 \%$. The magnetometer's estimate of the gyrofrequency was further improved by fine fitting of the $f_{\text {ce }}$ harmonics to the sounding data. The candidate plasma frequency values were tested in a separate fit. In contrast to the topside sounders, the best fit was sought for the series of Q-type resonances, $f_{\text {Qn }}$, using a weighted sum to favor the lowest orders of $f_{\mathrm{Qn}}$. Matching of $f_{\mathrm{Qn}}$ was found to be critically sensitive to the accuracy of the gyrofrequency determination, with errors of only $0.7 \%$ still causing misalignment of the fit. A similar matching concept was used in the resonance interpretation algorithm by Trotignon et al. [2001] for the Whisper relaxation sounder aboard the Cluster- 2 spacecraft. An amplitude envelope technique developed by Trotignon et al. [1986] had limited success, producing unacceptably high rates of false resonance recognitions.

[8] Considering the results of previous efforts, automated detection and identification of the resonance signatures in the RPI plasmagrams presents a great challenge because of the large range of plasma densities probed by RPI in the magnetosphere and the often less than optimal resolution of the frequency scans. The diversity of the scientific goals that the RPI targets along the orbit often results in a frequency range and resolution unfavorable for automatic detection of the key resonance signatures. Finally, no onboard magnetometer measurements are made to help constrain the $f_{\text {ce }}$ fitting procedure.

\section{A New Fitting Composite Resonance Model}

[9] Due to the specifics of the RPI plasmagrams, the automated fitting algorithm cannot rely on the guaranteed presence of any particular resonance on the plasmagram. RPI is a versatile instrument that adjusts its sounding frequencies along the spacecraft orbit to match changes in the plasma density and to meet a variety of scientific goals. Due to dynamic allocation of the frequencies from the operating band of $3 \mathrm{kHz}$ to $3 \mathrm{MHz}$ with either constant or logarithmically changing frequency steps ranging in size from $300 \mathrm{~Hz}$ to hundreds of $\mathrm{kHz}$, the RPI measurement parameters are often far from optimal to properly study the resonances. Compromises in the operating frequency band and resolution are commonly made to balance requirements for a high sounding cadence and long range coverage. Thus, plasmagrams may contain none of the $\mathrm{n} f_{\mathrm{ce}}$ resonances or more than 20 of them, and the frequency resolution may be fine enough to cover the resonance amplitude peak with several frequency steps, or be as coarse as to completely miss the resonance. The fitting scheme had to be adjusted to work for a varying number of resonances/harmonics that may be present on plasmagrams. The coarse frequency resolution effects were considered by determining whether the tested resonance is too far from the closest plasmagram frequency. As a result, the number of frequencies whose summary amplitudes contribute to the total fit quality becomes different for different sets of the driving $f_{\mathrm{ce}}$ and $f_{\mathrm{pe}}$. With a varying number of contributors at each fitting step, the fit quality cannot be calculated as the total sum, and therefore the "average fit" was used as the fit quality criterion:

$$
Q_{i j}=\frac{\sum_{\{f\}_{i j}} S_{1 R_{E}}(f)}{N},
$$

where $\{f\}_{i j}$ is subset of plasmagram frequencies $f$ corresponding to the model resonance frequencies calculated for the trial gyrofrequency $f_{c e}^{(i)}$ and the trial plasma frequency $f_{p e}^{(j)}$, such that each model frequency of the subset falls within the interval of $\left(f-\Delta_{\mathrm{r}}, f+\Delta_{\mathrm{r}}\right)$ of a plasmagram frequency $f$, where $\Delta_{\mathrm{r}}$ is the half-width of the expected frequency band taken by the resonance signature; $N$ is size of $\{f\}_{i j}$; and $S_{1 R_{E}}(f)$ is summary amplitude calculated over all ranges $r<1 \mathrm{R}_{\mathrm{E}}$ at the plasmagram frequency $f$, i.e.,

$$
S_{1 R_{E}}(f)=\sum_{r<1 R_{E}} A_{r}(f)
$$


where $A_{r}(f)$ is linear signal amplitude of the plasmagram bin $(r, f)$.

[10] For this fitting method to work, the true values of $f_{\mathrm{ce}}$ and $f_{\mathrm{pe}}$ have to be in the set of tested combinations, and the fit has to be robust to other data features such as traces and interference lines. The set of trial frequencies $f_{c e}^{(i)}$ and $f_{p e}^{(j)}$ is obtained by selecting low and high boundaries around the predicted model values of gyrofrequency $\left(f_{\mathrm{ce}}\right)_{\mathrm{p}}$ and plasma frequency $\left(f_{\mathrm{pe}}\right)_{\mathrm{p}}$. Then the frequency steps, $\Delta_{\text {ce }}$ and $\Delta_{\text {pe }}$, are selected that determine how many trial frequencies are selected from the interval around the model values.

[11] The Geopack Model of the Earth's magnetic field [Tsyganenko, 1990] is used to obtain $\left(f_{\mathrm{ce}}\right)_{\mathrm{p}}$. Most of the time, the interval of $\pm 5 \%$ around $\left(f_{\mathrm{ce}}\right)_{\mathrm{p}}$ is sufficient, except for periods of high magnetic activity at large radial distances from the Earth, when the interval is increased to $\pm 40 \%$. The prediction of the local plasma frequency $\left(f_{\mathrm{pe}}\right)_{\mathrm{p}}$ is even more uncertain, especially when the spacecraft is in the vicinity of the modeled plasmapause or magnetopause. The actual value may be one or two orders of magnitude off the modeled value. To make computational matters worse, the composite model appears to be sensitive to even a fraction of one per cent deviations of the input $f_{\mathrm{ce}}$ and $f_{\mathrm{pe}}$ from the true values, so that practically all plasmagrams require subpixel accuracy of the resonance signature localization (i.e., better than the frequency resolution of the plasmagram), and the trial frequency steps, $\Delta_{\text {ce }}$ and $\Delta_{\text {pe }}$, are forced to be as small as $0.1 \mathrm{kHz}$. The resulting computing time of the composite model fit becomes unacceptable in the framework of the interactive data analysis with the BinBrowser tool [Galkin et al., 2001], where the scaler starts the automated resonance matching and waits for its results.

[12] Splitting the composite model in two separate fits, as in Igi et al. [1982], reduced the computing time but led to unsatisfactory false recognition rates, primarily because of the sensitivity of the gyrofrequency fitting to the presence of other resonances, noise, and natural emission bands. Introduction of heuristics to improve noise resistance was attempted, but did not solve the problem. Even if there were no resonance signatures on the plasmagram, the model fit approach would still give a best fit solution. We therefore developed a method that is able to locate and match the resonance signatures when they are present as well as identify the lack of resonance signatures when they are absent.

\section{Detecting and Matching Plasmagram Resonances}

[13] Instead of fitting the composite model of resonances directly to a plasmagram image, the image is first analyzed to identify frequencies that are potential reso- nances, and then a matching algorithm is applied to classify these candidates as either a particular resonance type or a noise (interference) line.

\subsection{Resonance Detection}

\subsubsection{Smoothing}

[14] A great variety of noise reduction and signal enhancement techniques have been devised to improve the quality of signature detection in images. Ziou and Tabbone [1998] give a review of conventional image smoothing filters and arguments for their use. We designed a novel filter to improve the quality of resonance detection in plasmagrams, since our analysis of applicable classic 2D ridge detection filters [e.g., Subirana-Vilanova and Sung, 1992] showed that they tend to wash out and displace the subtle resonance signatures. The new 1D filter replaces each amplitude of the scan, $A_{\mathrm{r}}$, with the median calculated over the amplitudes with smaller virtual range,

$$
F\left(A_{\mathrm{r}}\right)=\operatorname{median}\left\{A_{\mathrm{j}}\right\}, j=[1, r] .
$$

[15] This concept is further referred to in the text as the "cumulative median". The cumulative median filter has a smoothing scale that gradually increases with range, so that it applies less smoothing at the lower ranges, preserving the resonance signature shape, and eventually arrives at a robust estimate of the average background level for that frequency. Besides protection of further processing stages from random noise and jitter, the cumulative median filter enhances a particular type of signature whose amplitude falls with range and suppresses all others. It is effectively matched to the expected shape of the resonance envelope that should have a falling slope due to eventual loss of the wave power. Figure 2 illustrates the response of the cumulative median filter to synthesized signals with rising and falling envelopes. The filter enhances the falling shape (Figure 2a) and reduces the rising slope (Figure 2b), thus improving the contrast of resonances in the plasmagram. Figure 3 demonstrates the noise-suppression performance of the filter applied to the same signal shapes with the addition of $30 \%$ white noise. In both cases the filter successfully removes the jitter and improves the contrast of the resonance.

[16] Figure 4 shows a sample RPI plasmagram obtained on June 28, 2001, at 23:58 UT before (Figure 4a) and after (Figure $4 \mathrm{~b}$ ) the filtering operation. Smoothing out the noise jitter and the remote echoes visually simplifies the resonance detection without compromising the accuracy of the frequency registration.

\subsubsection{Labeling}

[17] After applying the cumulative median filter, the next processing step is the resonance signature labeling 


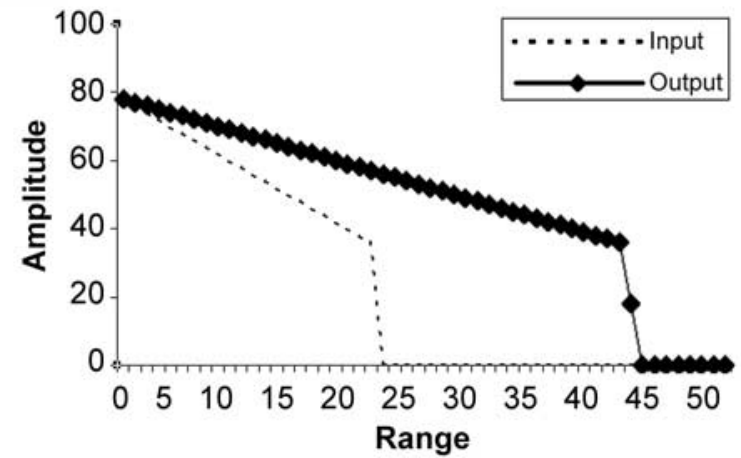

(a)

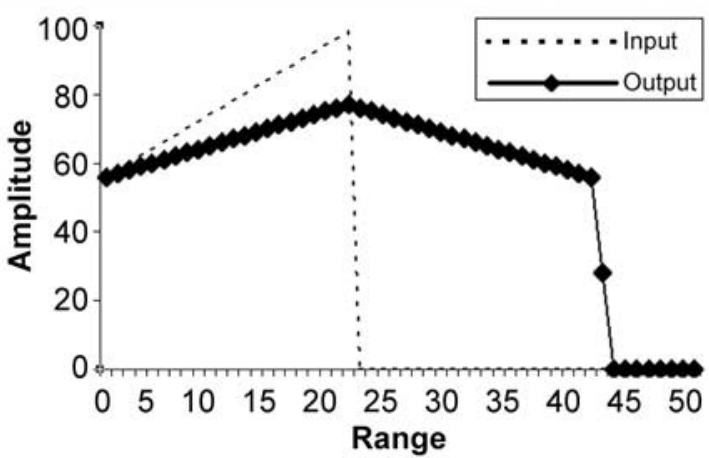

(b)

Figure 2. Response of the cumulative median filter to synthesized signals with falling (a) and rising (b) envelopes. The filter elevates the falling envelope that the resonance signatures are expected to display and reduces the rising slope, thus improving the signature contrast.

that tags the frequencies containing resonances using pattern recognition methods. A two-step labeling procedure is used, where frequencies that contain no resonances are first excluded, and then frequency groups are identified that belong to the same resonance. A frequency is excluded from the resonance analysis if its amplitude envelope does not display the appropriate decay with time. This is determined by evaluating the slope of the straight line fitted to the envelope using the leastsquare fitting technique. This procedure is used together with receiver saturation criteria that prevent elimination of strong resonances that do not show the expected amplitude decay. Saturation artifacts are occasionally observed for measurements with high receiver gains. At any particular fixed base gain, the system dynamic range is determined by the bit resolution of the voltage digitizer $(12$ bits $=72 \mathrm{~dB})$ and choice of 4,8 , and 16-chip phase code waveforms that can add up to $24 \mathrm{~dB}$ of dynamic range after pulse compression. The total dynamic range then varies from $72 \mathrm{~dB}$ (plain pulse waveform, fixed gain) to $126 \mathrm{~dB}$ (16 chip waveform, $30 \mathrm{~dB}$ range of the autogain adjustment). Commonly the resonance study plasmagrams are made at a lower dynamic range setting: (a) pulse compression is not used as it suppresses the plasma waves that do not have appropriate phase code, and (b) the autogain evaluation

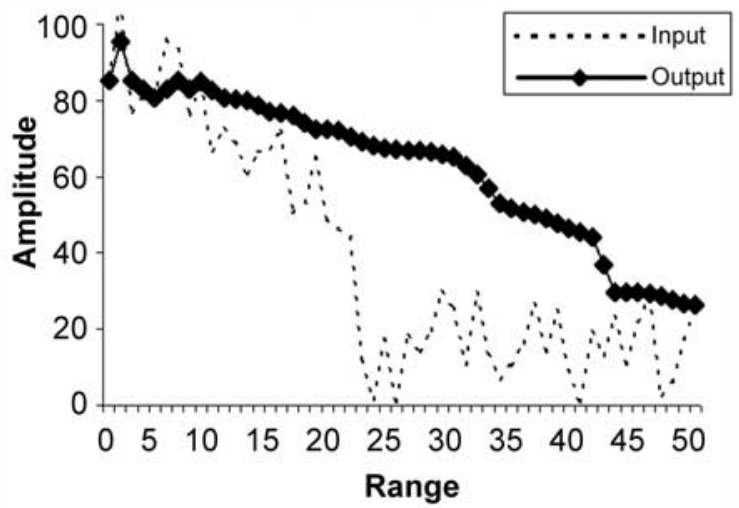

(a)

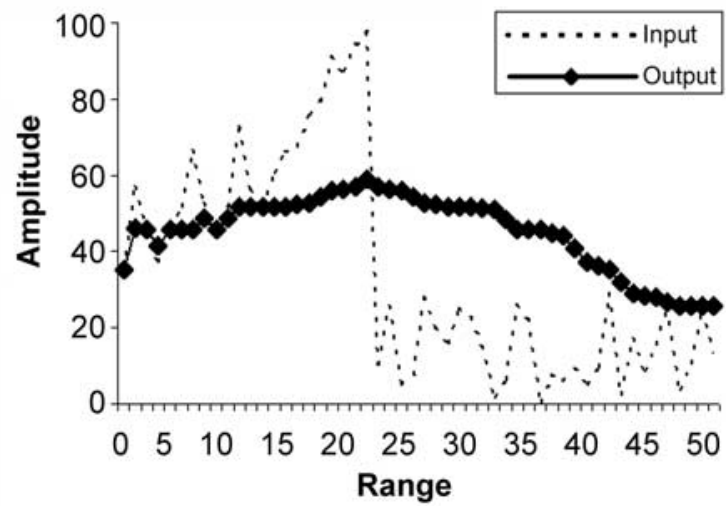

(b)

Figure 3. Response of the cumulative median filter to synthesized signals with falling (a) and rising (b) envelopes, with the addition of $30 \%$ noise. The filter smoothes the noise and enhances the contrast of the resonance signatures. 


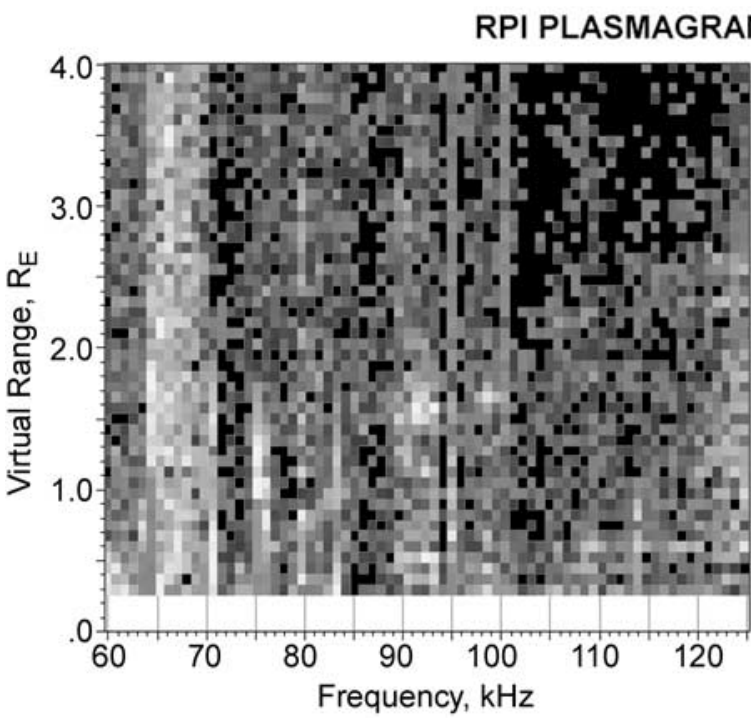

(a)

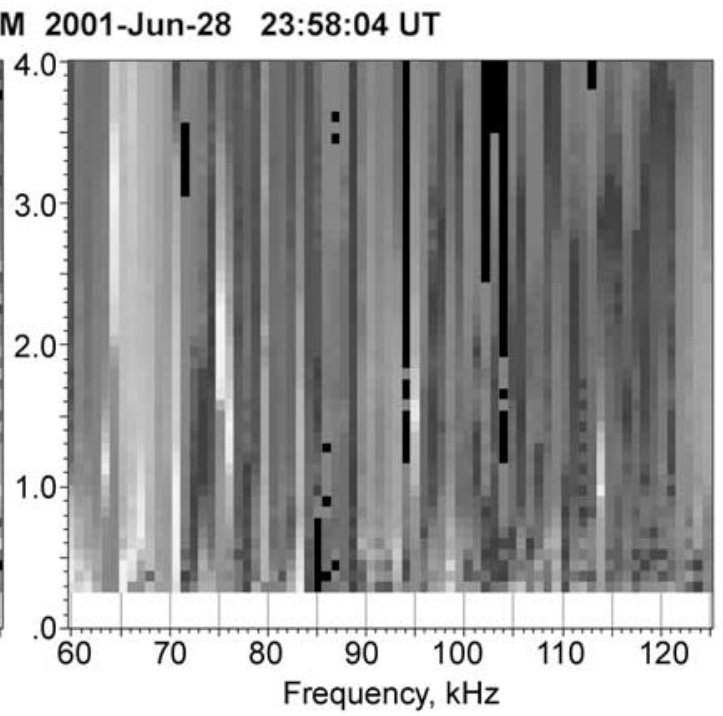

(b)

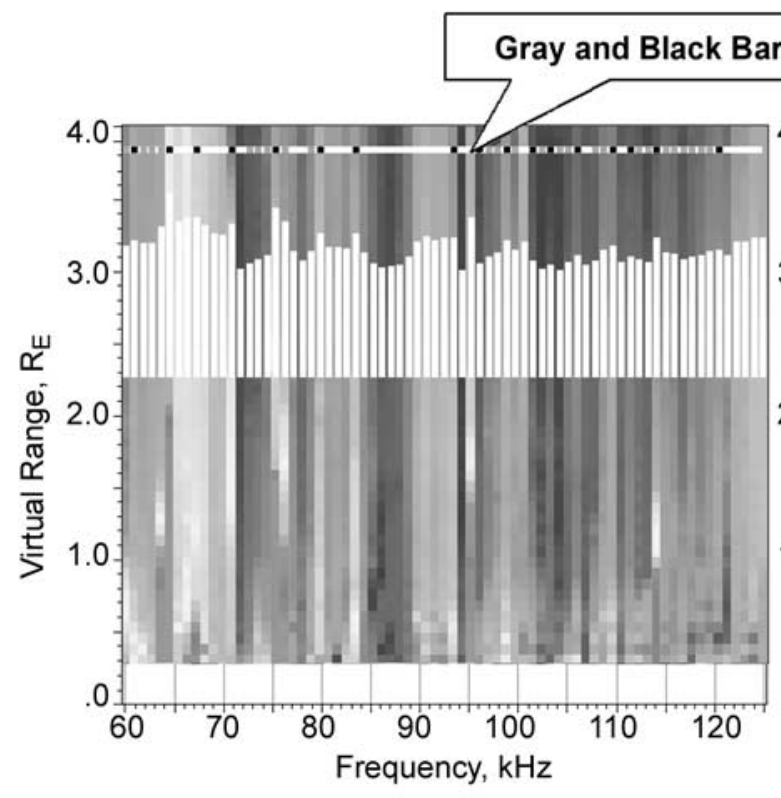

(c)

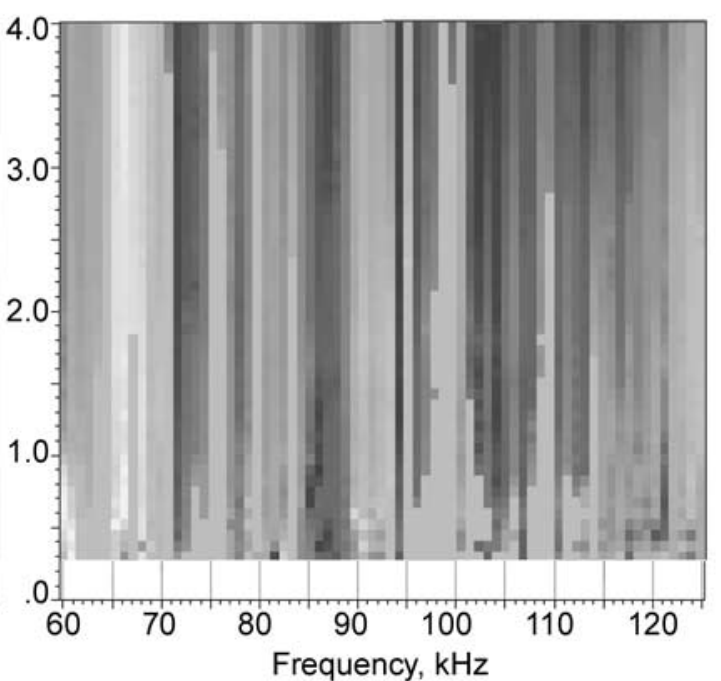

(d)

Figure 4. Resonance detection in RPI plasmagrams. (a) Raw plasmagram. (b) Processing with the cumulative median filter. (c) Summary amplitude function (white bars), labeled frequencies containing resonances (gray bars) and detected peaks (black bars). (d) Localized resonance signatures. See color version of this figure at back of this issue.

is disabled as it requires an additional $200 \mathrm{~ms}$ per frequency that becomes a considerable overhead for the resonance study measurements. To avoid loosing resonances due to the saturation we do not exclude any frequencies where $90 \%$ of the amplitudes are above the saturation threshold.

[18] After exclusion of all frequencies without resonance signatures, the frequency intervals belonging to 

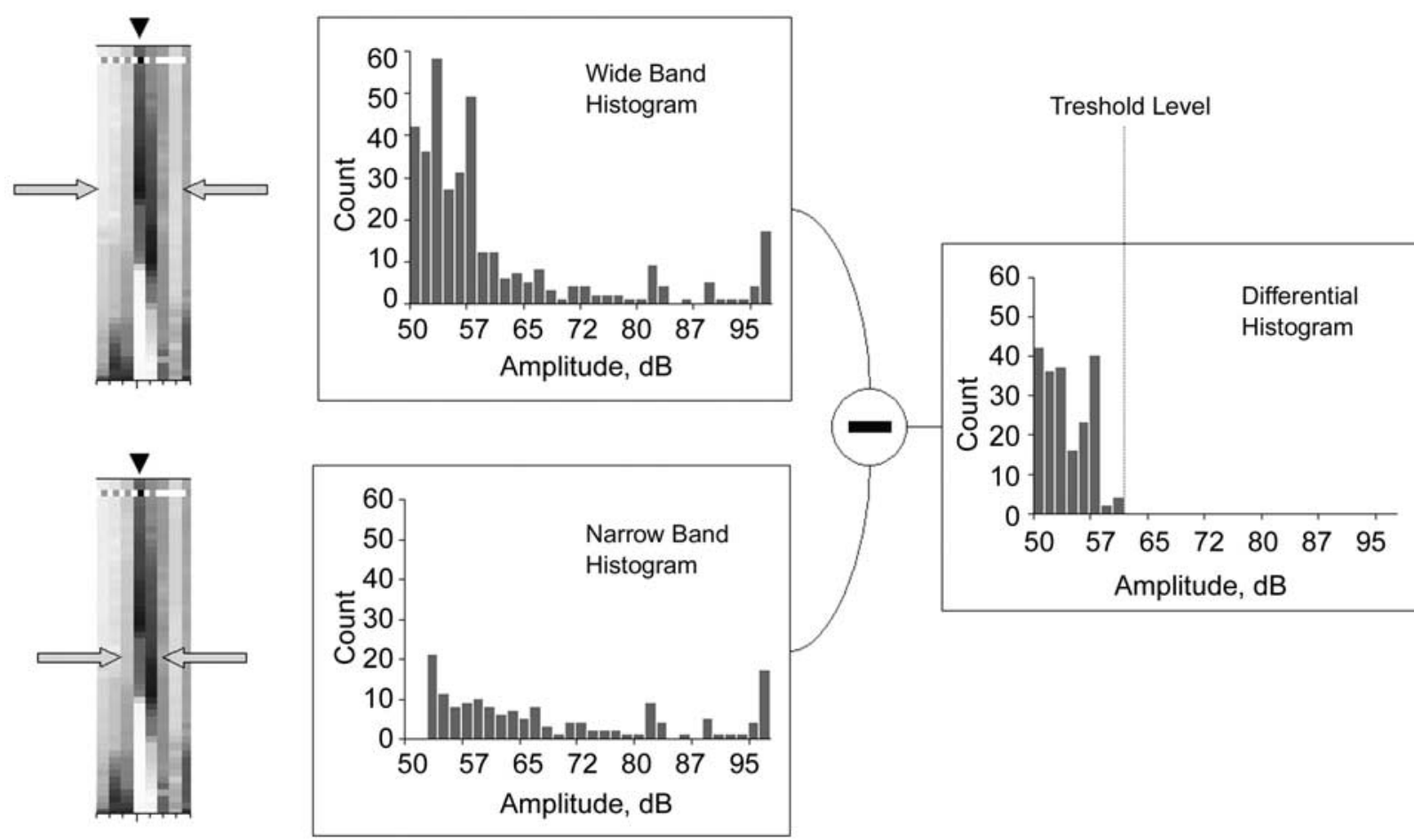

Figure 5. Use of the differential histogramming technique to estimate the local detection threshold for the resonance signature. See color version of this figure at back of this issue.

the same resonance signatures are determined by collapsing the image intensities along the vertical axis and seeking the peaks of the resulting summary amplitude function, $\mathrm{S}(f)$. The status line on top of the plasmagram in Figure $4 \mathrm{c}$ shows valid frequencies in gray, and the peaks identified in black.

\subsubsection{Contrast Evaluation}

[19] To further improve the robustness of the matching algorithm, a measure of contrast is calculated for each signature identified, so that stronger signatures contribute more to the fit quality. To determine the contrast, the average amplitude of the signature is compared to the average background amplitude. We use a statistical technique of differential histogramming to distinguish resonances from the background. In Figure 5, the lower amplitude histogram is built using all the amplitudes from all the range bins in each frequency step contained in a narrow $\pm 1 \mathrm{kHz}$ band around the resonance peak frequency, and the upper histogram is calculated over a wider $\pm 2.5 \mathrm{kHz}$ band that is guaranteed to include both resonance and background noise. Subtracting histogram 1 from histogram 2 leaves only background amplitudes, and their upper boundary defines the amplitude threshold for the resonance detection. Figure $4 \mathrm{~d}$ shows in magenta the amplitudes exceeding the threshold. After the signature is localized, its contrast against the background is calculated by averaging the gradients between the tagged amplitudes and their immediate neighbors.

[20] The resonance detection procedure labels a subset of plasmagram frequencies as potentially belonging to resonances, and preliminarily determines location and contrast of the resonance signatures. This information enters the interpretation algorithm that matches the frequencies to their theoretical counterparts. The matching starts with identification of the resonances produced at the gyrofrequency and its harmonics. Without knowledge of the actual value of $f_{\text {ce }}$, further interpretation of the plasmagram resonances is impossible.

\subsection{Matching the Gyrofrequency Resonances}

[21] At this point of the analysis, a list of detected resonance-like signatures and a predicted value of the gyrofrequency, $\left(f_{\text {ce }}\right)_{\mathrm{p}}$, exist. The task is to find $\mathrm{n} f_{\mathrm{ce}}$ resonances in the list and thus determine the actual value of $f_{\text {ce. }}$. Again, we can try a number of trial $f_{c e}^{(i)}$ values around the predicted value and see which one produces the best match. This fit is different from the previously considered composite or separate model fitting tasks in that the pattern recognition analysis has provided the actually detected resonance signatures. Only those frequencies contribute to the fit that are labeled as containing a resonance, and the resonances of higher contrast contribute more to the fit quality. 


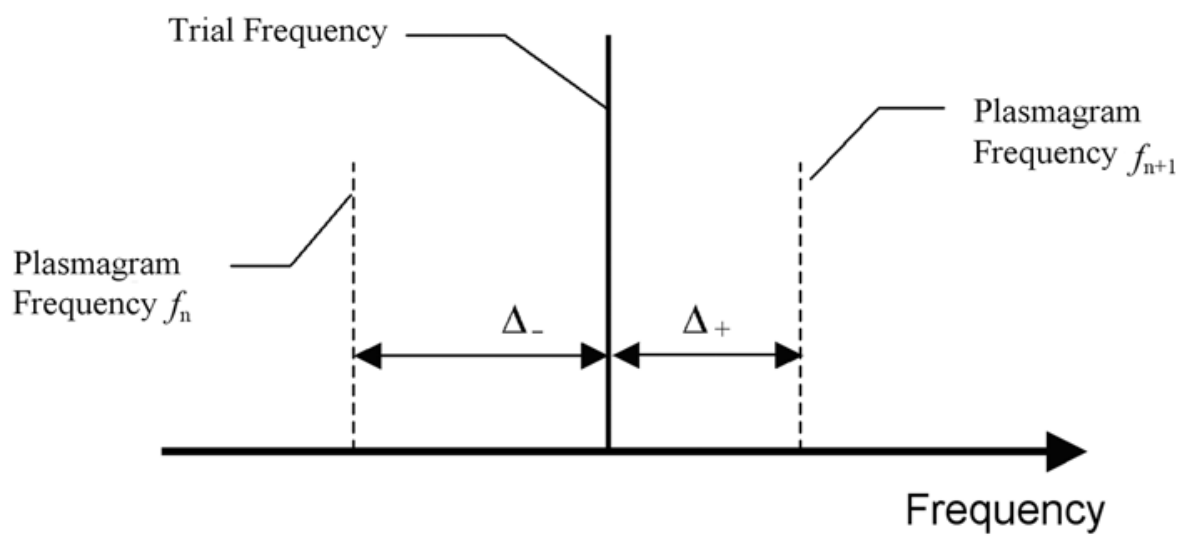

Figure 6. Choice of the actual plasmagram frequencies next to the trial frequency. If the closest frequency is not tagged as containing a resonance, the other frequency contributes to the fit quality at a reduced weight.

[22] Analysis of the RPI plasmagrams for the gyrofrequency harmonics showed that they do not always match perfectly to multiples of $f_{\text {ce }}$. One of the causes of this mismatch is the change of spacecraft location, and therefore $f_{\text {ce }}$, within the time needed to acquire the plasmagram, which is typically $1-3 \mathrm{~min}$. The fitting scheme was modified to correct the higher orders of $n f_{c e}$ for the expected change in the magnetic field strength as the spacecraft changes its location. Another consideration was given to possible localization errors and to insufficient frequency resolution that prevents separation of two neighboring resonances. Figure 6 illustrates the general principle of selecting matching signatures next to a trial frequency, where first preference is given to the closer actual plasmagram frequency. If, however, the closest frequency is not identified as valid (i.e., containing a resonance), the other actual frequency, if valid, is allowed to contribute to the fit at a lower weight. Both plasmagram frequencies are tested if they are still within the expected frequency band occupied by the resonance through analysis of their deviations from the trial frequency, $\Delta_{+}$and $\Delta_{-}$. This sentence is not clear.

\subsection{Matching the Plasma Frequency Resonance}

[23] Fitting of the plasma frequency is affected by the same problems discussed above for the gyrofrequency fit: (a) insufficient frequency coverage and resolution, (b) changes in the medium during the plasmagram measurement time, (c) need for subpixel accuracy of the frequency determination, and (d) prediction errors. In addition to these common issues, the model equations describing dependencies of the resonances on the plasma frequency, such as equation (2) for the Q-type resonance frequencies, do not always apply to the plasma in the magnetosphere that may contain a hot component in addition to the (dominant) cold population. In a plasma that is not described by a Maxwellian distribution, the best match of Q type resonances based on equation (2) will yield $f_{\mathrm{pe}}$ and $f_{\mathrm{uh}}$ values that are not likely to match any of the resonances in the plasmagram [Benson et al., 2003]. Our present algorithm does not include a match of resonances to the Dn frequencies given by (3).

[24] Figure 7 shows examples of fully automated resonance processing with the developed algorithm. The values are compared with predicted fce values based on the Tsy 96-1 magnetic-field model [Tsyganenko, 1996] and the ad-hoc $f_{\text {pe }}$ model used with the RPI data [Reinisch et al., 2001]. For the three examples, all resonance signatures were recognized and matched correctly. The modeled $f_{\text {ce }}$ values agreed closely with the measured values, whereas large differences between the predicted and observed values were detected for $f_{\text {pe, }}$ signifying that the plasma conditions were far from expected. As a result, $f_{\text {uh }}, f_{\mathrm{z}}$, and $f_{\mathrm{x}}$ are very different from the model. The high frequency resolution of the plasmagram in the central panel allowed detecting an approximate $1 \%$ overshoot of the model Qn resonances, which is comparable to the observational uncertainty but

Figure 7. Examples of automated resonance matching. The upper panel shows raw plasmagrams; the middle row displays results of accumulative median filtering operation and places markers at automatically scaled resonance and cutoff frequencies. $P=f_{\text {pe }}, T=f_{\text {uh }}, x=f_{x}$ (yellow arrow), $z=f_{z}$ (purple arrow), $1,2 \ldots=n f_{c e}, Q n=f_{\text {Qn }}$. Lower panel gives autoscaled frequencies in comparison with the predicted values. See color version of this figure at back of this issue. 

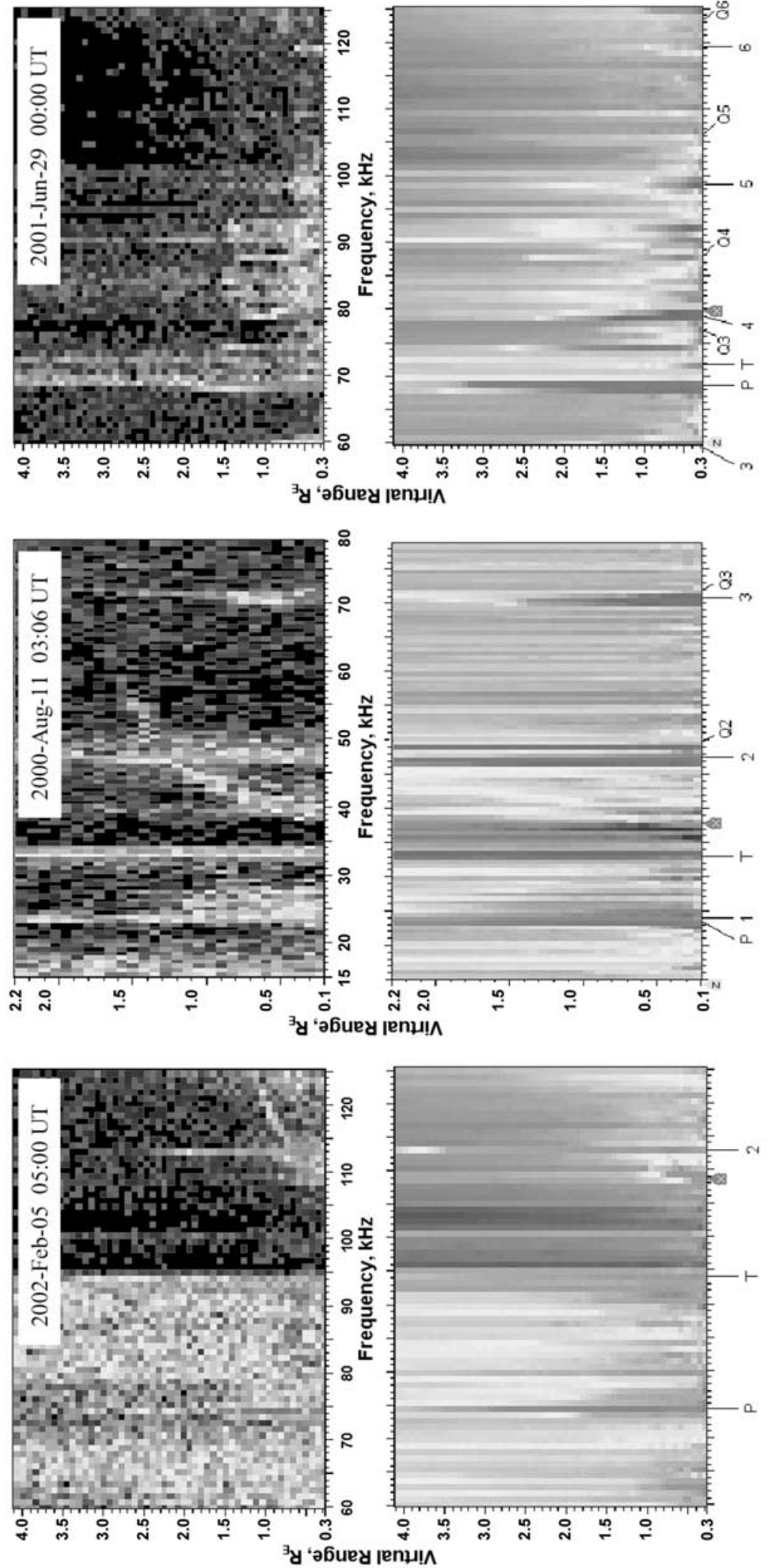

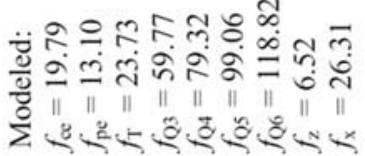

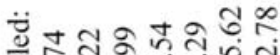

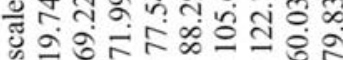

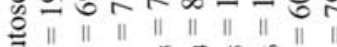



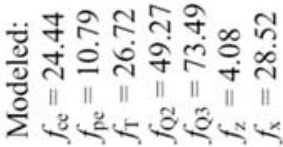

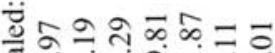

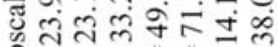

อ || || || || || ||

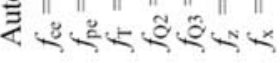



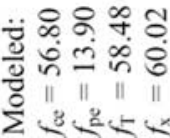

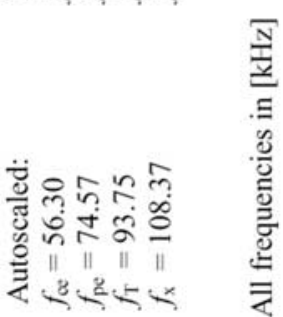


may also be caused by deviations of the plasma distribution from the Maxwellian assumed in the expressions used for Qn.

\section{Discussion of Results}

[25] The three major features that led to successful automated interpretation of the resonances are (a) use of pattern recognition techniques to detect and evaluate resonance signatures prior to matching, (b) accounting for changes in the medium during the time required to acquire a plasmagram, and (c) use of the cumulative median filter for enhancement of the resonance signatures. The new algorithm now correctly and reliably interprets a wide range of scenarios found in RPI plasmagrams. Whereas the overall quality of gyrofrequency automatching is satisfactory, it is often difficult to correctly identify the plasma frequency resonance. In the case of the former, there is a harmonic sequence that aids the autodetection algorithm and there is a fairly reliable model to constrain the search criteria; in the case of the later, neither is available. While the observed magnetic-field strength can be higher than the quietcondition model by tens of percent on disturbed days, the deduced electron density can be higher than the model by many factors of ten (R. F. Benson et al., manuscript in preparation, 2004).

[26] Mismatches fall into three general categories: (1) no match can be found because the medium gradients are different from predicted ones; (2) key signature(s) are either outside the plasmagram coverage or they are obscured; (3) a false match is selected due to errors in the resonance signature detector.

\subsection{Errors in Prediction of Medium Gradients}

[27] Noticeable mistakes in predicting the general gradient of gyrofrequency, $\Delta f_{\text {ce, }}$, are very rare. It is more common to observe natural fluctuations in the magnetic field that cause occasional mismatches of $f_{\text {ce }}$ harmonics in plasmagrams taken with high frequency resolution. The frequency bin selection technique discussed in Section 3.2 allows mismatched harmonics to still contribute to the quality of the fit if their deviation does not exceed one frequency step. The gradient of plasma frequency, $\Delta f_{\text {pe }}$, is frequently predicted incorrectly in the vicinity of the plasmapause because of the difficulty in accurately modeling the location and gradient of this boundary.

\subsection{False Decisions by the Signature Detector}

[28] While selecting plasmagram frequencies as resonances based on the amplitude decay pattern (section 3.1.2) discriminates against interference lines, it can also eliminate resonances that do not display any significant amplitude decay over the listening time interval sampled in the plasmagram. It is difficult to select the tagging criterion optimally so as to avoid both false positive and false negative decisions; the current choice is to allow false "valid" frequencies to enter the matching phase rather than to remove good signatures. Although the contribution of such false frequencies to the fit is typically small because of their low contrast against the background, they still increase the likelihood of wrong matches.

\subsection{Key Resonance Signatures Missing or Obscured}

[29] The automatching algorithm works best when there are a few distinct resonance signatures present in the plasmagram so that the fit quality maximizes when the model and the measurements match. If the frequency coverage is not sufficient to include enough signatures in the plasmagram, the best fit may not correspond to the correct answer. Also, when the $\mathrm{n} f_{\text {ce }}$ and $f_{\mathrm{Qn}}$ separation is not significant (for low ratios of $f_{p e} / f_{c e}$ ) the matching may not be optimal because it relies completely on the proper identification of $f_{\mathrm{pe}}$ and $f_{\mathrm{uh}}$ which can fall outside the frequency coverage or can be overlapped by other resonances. Proper interpretation of such cases often requires analysis of the previous and following plasmagrams to confirm changing patterns as plasma conditions change. Such an analysis, as well as the inclusion of the Dn frequencies in the matching routine, remains to be done.

\section{Conclusion}

[30] Detection and interpretation of the resonances in RPI plasmagrams stands as an important diagnostic tool and a necessary step towards further interpretation of the remote sensing information contained in these records. Automation of this procedure relieves the RPI data analyst from tedious calculations. The method described in this paper presents a novel approach to automated resonance detection and interpretation in a plasma environment with large variability like the one encountered by the RPI instrument on IMAGE. Implementation of this approach into the BinBrowser data visualization and analysis tool [Galkin et al., 2001] has made it possible to automatically scale all of the approximately 600 plasmagrams recorded every day by RPI. The algorithm could also become a useful tool for onboard processing of future active plasma-wave instruments.

[31] Acknowledgments. The work at the University of Massachusetts Lowell was supported by NASA under Southwest Research Institute subcontract 83822, and through the Intelligent Systems (IS) grant NAG5-13387.

\section{References}

Benson, R. F., V. A. Osherovich, J. Fainberg, A. F. Vinas, and D. R. Ruppert (2001), An interpretation of banded magneto- 
spheric radio emissions, J. Geophys. Res., 106, 13,17913,190 .

Benson, R. F., V. A. Osherovich, J. Fainberg, and B. W. Reinisch (2003), Classification of IMAGE/RPI-stimulated plasma resonances for the accurate determination of magnetospheric electron-density and magnetic field values, J. Geophys. Res., 108(A5), 1207, doi:10.1029/ 2002JA009589.

Burch, J. L. (Ed.) (2000), The IMAGE Mission, 506 pp, Kluwer Acad., Norwell, Mass.

Galkin, I. A., G. M. Khmyrov, A. Kozlov, B. W. Reinisch, X. Huang, and G. Sales (2001), New tools for analysis of space-borne sounding data, paper presented at National Radio Science Meeting, Union Radio Sci. Int., Boston, Mass.

Huang, X., and B. W. Reinisch (1982), Automatic calculation of electron density profiles from digital ionograms: 1. Automatic $\mathrm{O}$ and $\mathrm{X}$ trace identification for topside ionograms, Radio Sci., 17, 421-434.

Huang, X., B. W. Reinisch, D. Bilitza, and R. F. Benson (2002), Electron density profiles of the topside ionosphere, Ann. Geophys., 45, 125-130.

Igi, S., K. Aikyo, and R. Nishizaki (1982), Efficient coding and resonance spike identification for topside ionogram processing, Mem. Natl. Inst. Polar Res. Spec. Issue, 22, 140-154.

Muldrew, D. B. (1972), Electron resonances observed with topside sounders, Radio Sci., 7, 779-789.

Osherovich, V. A., and R. F. Benson (1991), The lower subsidiary diffuse plasma resonances and the classification of radio emissions below the plasma frequency, J. Geophys. Res., 96, 19,331-19,341.

Pulinets, S. A., and R. F. Benson (1999), Radio frequency sounders in space, in Review of Radio Science, edited by W. R. Stone, pp. 711-733, Oxford Univ. Press, New York.

Reinisch, B. W. (1996), Modern ionosondes, in Modern Ionospheric Science, pp. 440-458, Eur. Geophys. Soc., London.

Reinisch, B. W., et al. (2000), The Radio Plasma Imager investigation on the IMAGE spacecraft, Space Sci. Rev., 91, $319-359$.
Reinisch, B. W., et al. (2001), First results from the Radio Plasma Imager on IMAGE, Geophys. Res. Lett., 28 , $1167-1170$.

Stix, T. H. (1962), The Theory of Plasma Waves, 283 pp., McGraw-Hill, New York.

Subirana-Vilanova, J. B., and K. K. Sung (1992), Multi-scale vector-ridge-detection for perceptual organization without edges, A.I. Memo 1318, 48 pp., Artif. Intel. Lab., Mass. Inst. of Technol., Cambridge.

Trotignon, J. G., J. Etcheto, and J. P. Thouvenin (1986), Automatic determination of the electron density measured by relaxation sounder on board ISEE 1, J. Geophys. Res., 91, 4302-4320.

Trotignon, J. G., et al. (2001), How to determine the thermal electron density and the magnetic field strength from the Cluster/Whisper observations around the Earth, Ann. Geophys., 19, 1711-1720.

Tsyganenko, N. A. (1990), Quantitative models of the magnetospheric magnetic field: Methods and results, Space Sci. Rev., 54, 75-186.

Tsyganenko, N. A. (1996), Effects of the solar wind conditions on the global magnetospheric configuration as deduced from data-based field models, in Proceedings of $3 \mathrm{rd}$ International Conference on Substorms (ICS-3), Versailles, France, Eur. Space Agency Spec. Publ., ESA-SP 389,181-185.

Warren, E. S., and E. L. Hagg (1968), Observation of electrostatic resonances of the ionospheric plasma, Nature, 220, $466-468$.

Ziou, D., and S. Tabbone (1998), Edge detection techniquesAn overview, Int. J. Pattern Rec. Image Anal., 8, 537-559.

R. F. Benson, Laboratory for Extraterrestrial Physics, NASA Goddard Space Flight Center, Greenbelt, MD 20771, USA.

S. F. Fung, Space Physics Data Facility, Code 632, NASA Goddard Space Flight Center, Greenbelt, MD 20771, USA.

I. A. Galkin, X. Huang, and B. W. Reinisch, Center for Atmospheric Research, University of Massachusetts, 600 Suffolk Street, Lowell, MA 01854, USA. (ivan_galkin@uml. edu) 


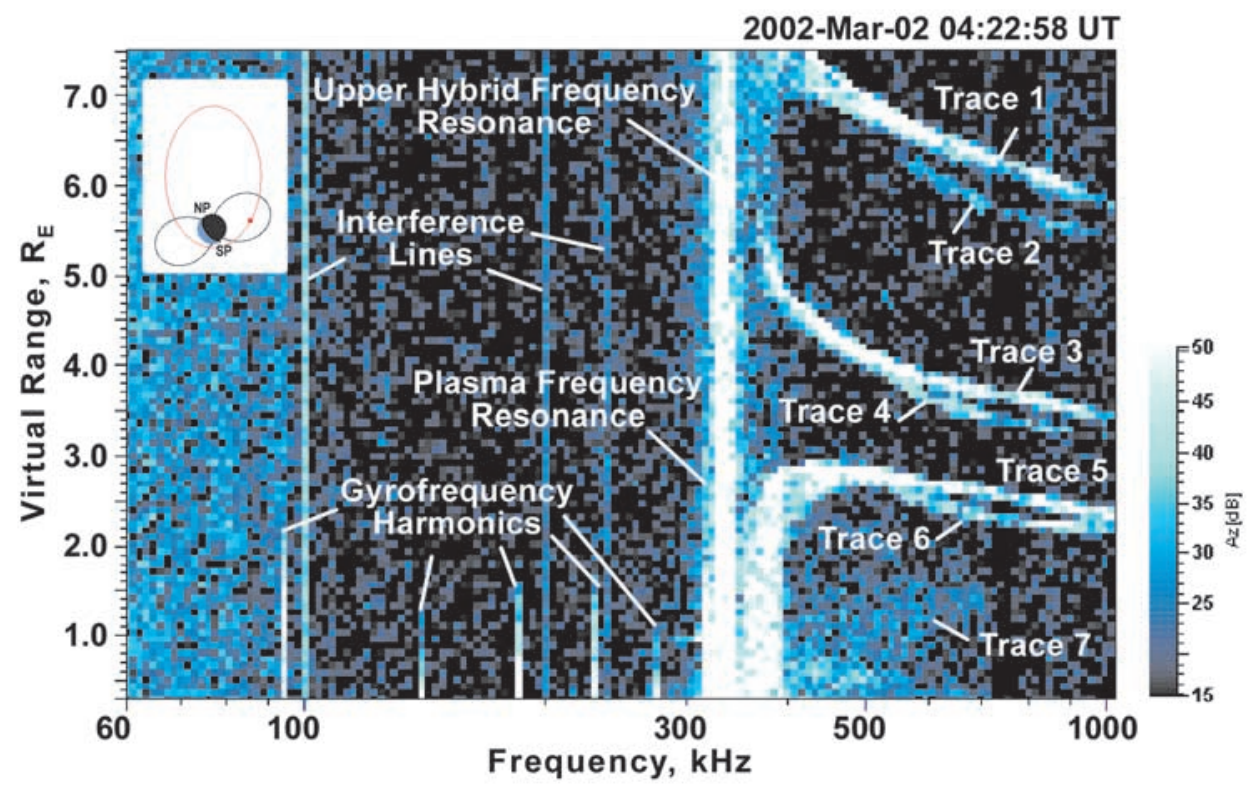

Figure 1. RPI Plasmagram on 2 March 2002, 04:23 UT with remote echo traces and resonance signatures as indicated. 




(a)

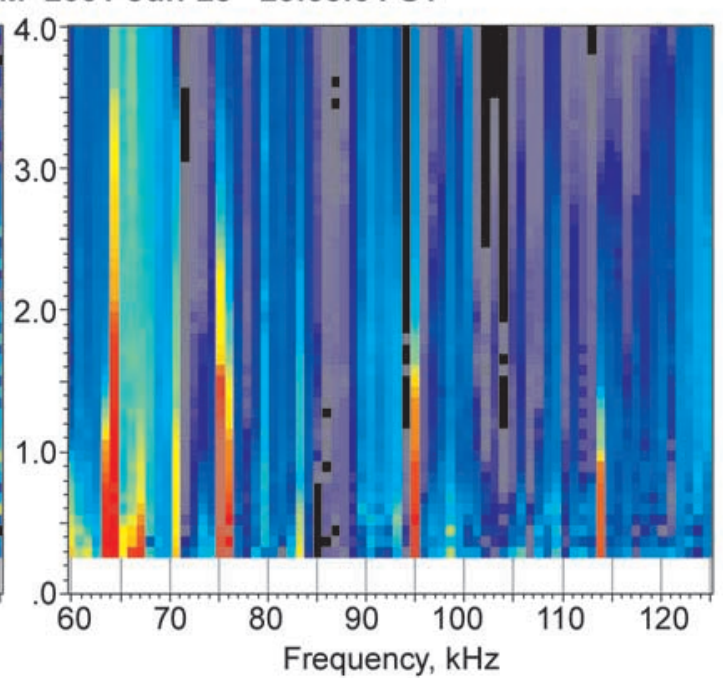

(b)

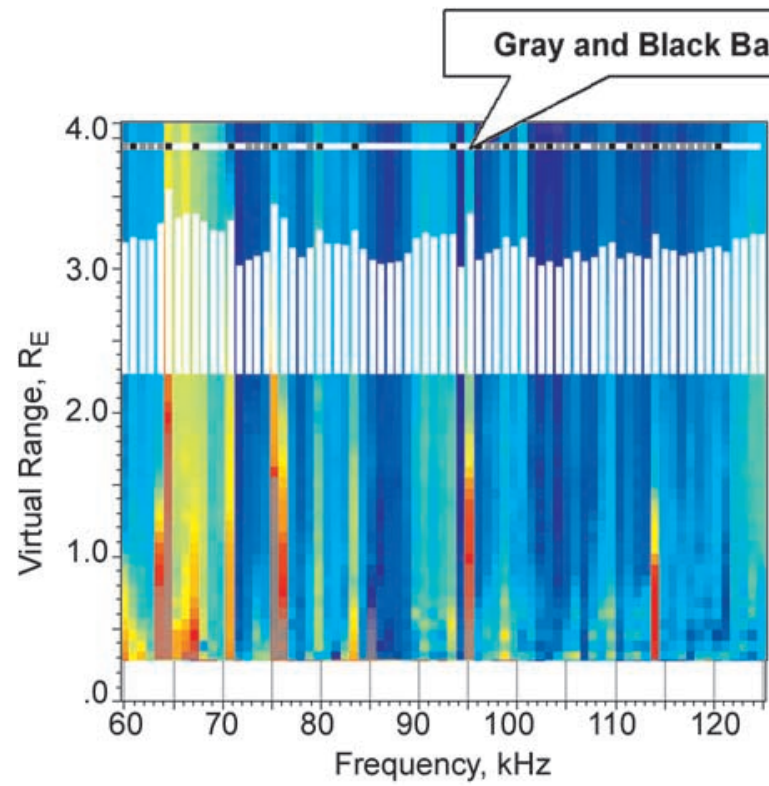

(c)

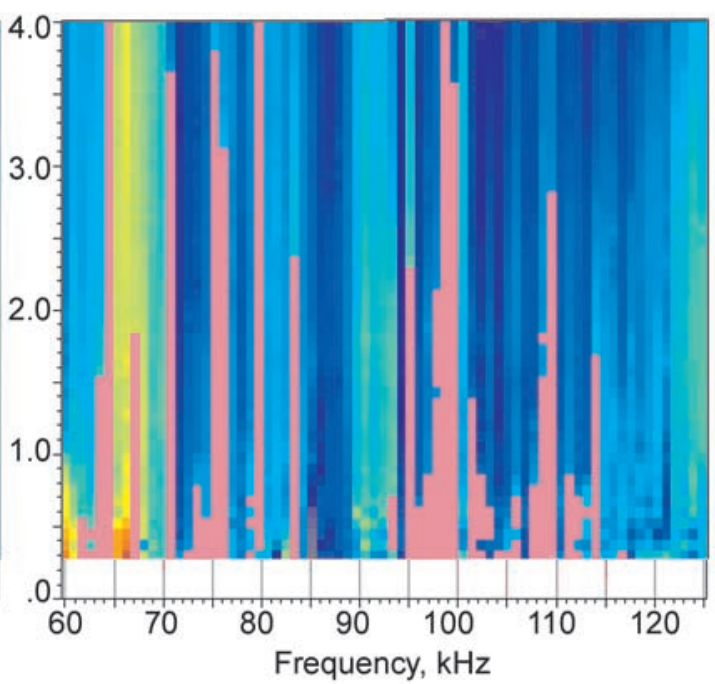

(d)

Figure 4. Resonance detection in RPI plasmagrams. (a) Raw plasmagram. (b) Processing with the cumulative median filter. (c) Summary amplitude function (white bars), labeled frequencies containing resonances (gray bars) and detected peaks (black bars). (d) Localized resonance signatures. 

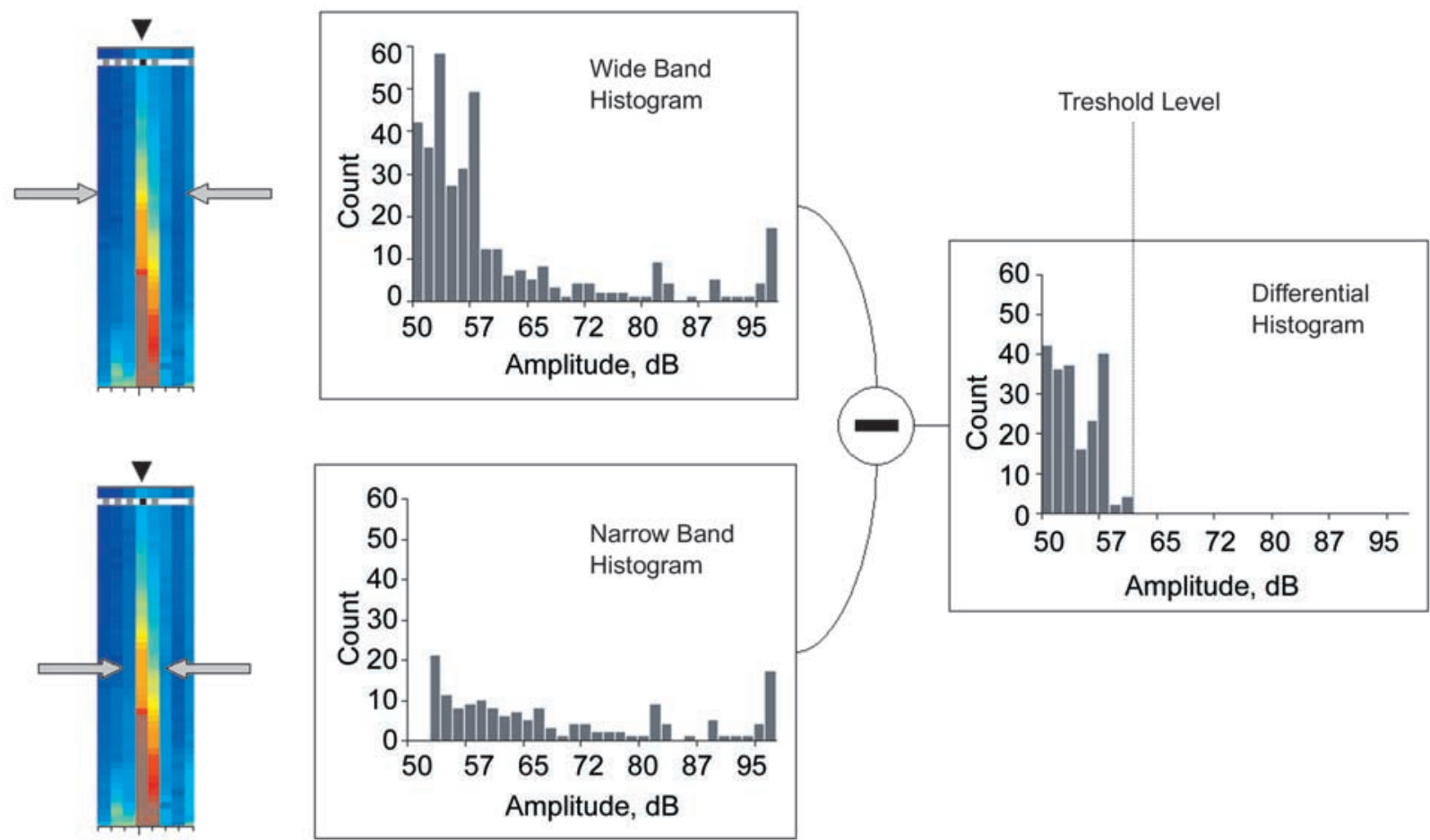

Figure 5. Use of the differential histogramming technique to estimate the local detection threshold for the resonance signature.

Figure 7. Examples of automated resonance matching. The upper panel shows raw plasmagrams; the middle row displays results of accumulative median filtering operation and places markers at automatically scaled resonance and cutoff frequencies. $P=f_{\text {pe }}, T=f_{\text {uh }}, x=f_{x}$ (yellow arrow), $z=f_{z}$ (purple arrow), $1,2 \ldots=n f_{\text {ce }}, Q n=f_{Q n}$. Lower panel gives autoscaled frequencies in comparison with the predicted values. 

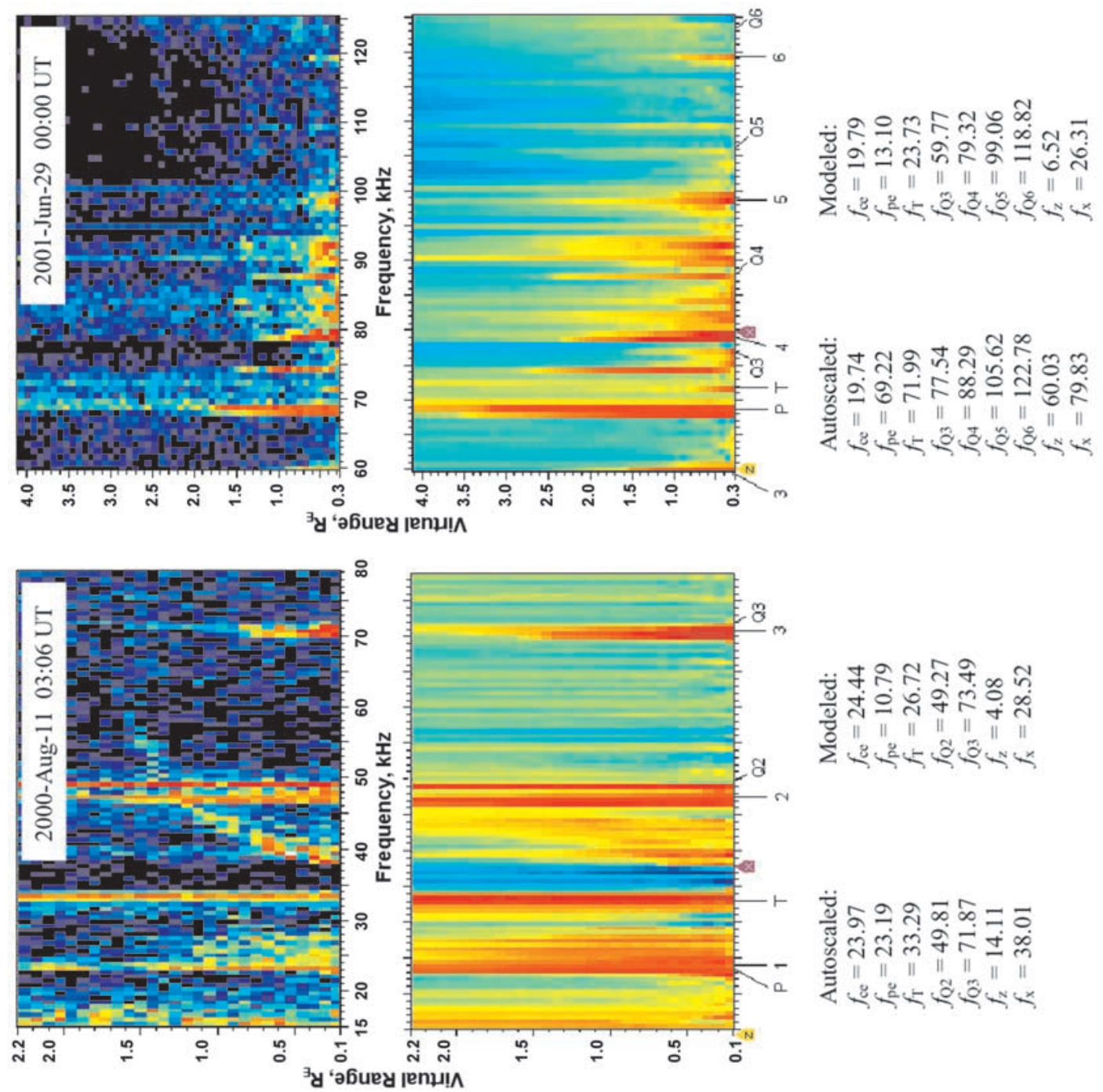

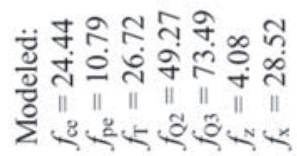

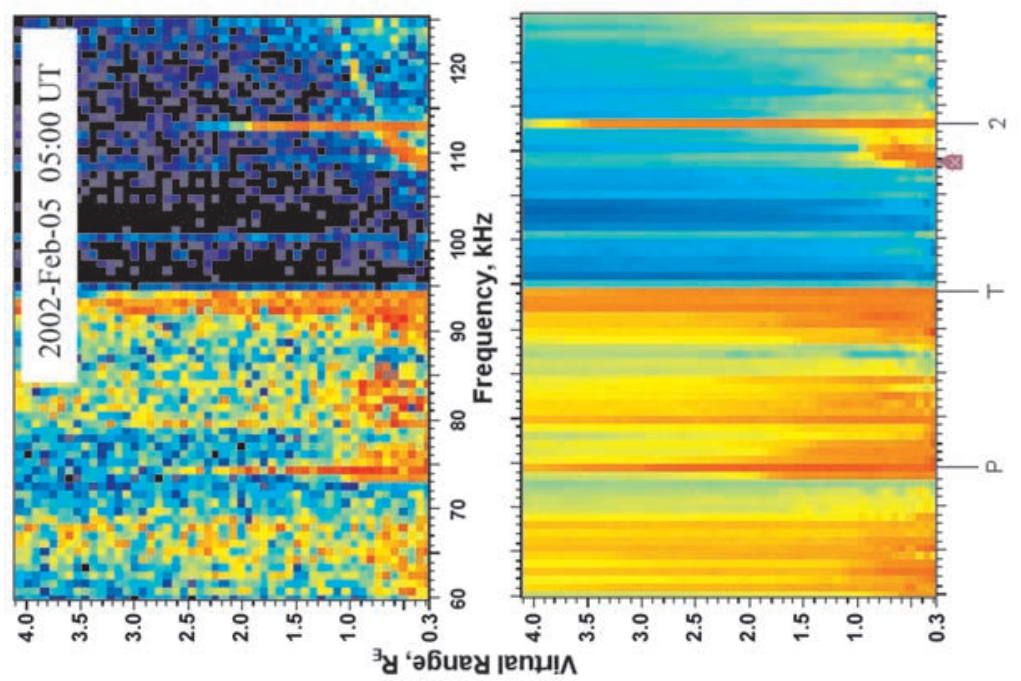

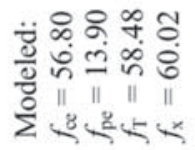

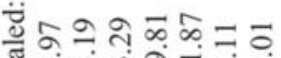

ฮู่

॥ || || || || || ||

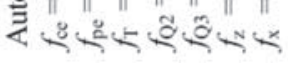

실 